\title{
La valutazione di Impatto Sanitario (VIS)
}

\author{
Vincenzo Russo ${ }^{1}$ and Virgilio Ciccone ${ }^{1}$ \\ ${ }^{1}$ Affiliation not available
}

\begin{abstract}
La Valutazione di Impatto sulla Salute (VIS) è una metodologia di valutazione che, assieme ad altri strumenti, permette di valutare gli effetti che determinati interventi possono produrre sulla salute umana e sull'ambiente, al fine di individuare eventuali impatti positivi e/o negativi. Questa ha dunque il ruolo di collegare il tema della salute, che costituisce un diritto fondamentale, con quello ambientale, poiché permette di valutare i rischi di un progetto sulla salute umana.
\end{abstract}

\section{Introduzione al quadro normativo e alla proce- dura di VIS}

Il percorso di elaborazione riguardo alla VIS inizia in Europa nei primi anni novanta con il Trattato di Maastricht del 1992 e con la sua evoluzione, rappresentata dal Trattato di Amsterdam del 1997, in entrambi infatti si pone l'accento sulle questioni riguardanti la tutela della salute umana e dell'ambiente e sullo sviluppo sostenibile ${ }^{1}$. La nascita vera e propria della VIS si colloca però all'interno del processo di evoluzione della VIA e di introduzione, attraverso la Direttiva 2001/42/CE, della VAS, caratterizzata da un processo valutativo integrato che affronta diverse tematiche, tra cui appunto quella della salute. In seguito, con la Direttiva 2003/35/CE, si garantisce poi la partecipazione del pubblico nei processi decisionali in campo ambientale ${ }^{2}$. II tema della tutela della salute, inoltre, viene affrontato anche nel Trattato di Kiev del 2003, dove si pone l'attenzione alla riduzione delle minacce ambientali sulla salute ${ }^{3}$.

Ad oggi, in Italia, esiste una sola legge nazionale sulla VIS, la n.221 del 28 Dicembre 2015 (art.9), che ha sì introdotto la procedura di Valutazione di Impatto sulla Salute nell'ambito dei procedimenti di VIA statale, ma esclusivamente per progetti riguardanti le raffinerie di petrolio greggio, gli impianti di gassificazione e liquefazione, le centrali termiche e gli altri impianti di combustione con potenza termica superiore a $300 \mathrm{MW}^{4}$. 
Il concetto di salute su cui si basa tutto il procedimento di VIS è quello definito dall'OMS nel 1948, secondo cui "la salute è uno stato di completo benessere fisico, sociale e mentale, e non soltanto l'assenza di malattia o di infermità", che fa riferimento ai cosiddetti determinanti di salute, ossia fattori biologici naturali, comportamenti e stili di vita, ambiente fisico e sociale, possibilità di accesso alle cure sanitarie e a servizi in genere 5 . Il compito della VIS è proprio quello di valutare come un intervento sul territorio possa indurre cambiamenti, anche non intenzionali, su questi determinanti e, di conseguenza, produrre un cambiamento nello stato di salute della popolazione esposta ${ }^{6}$.

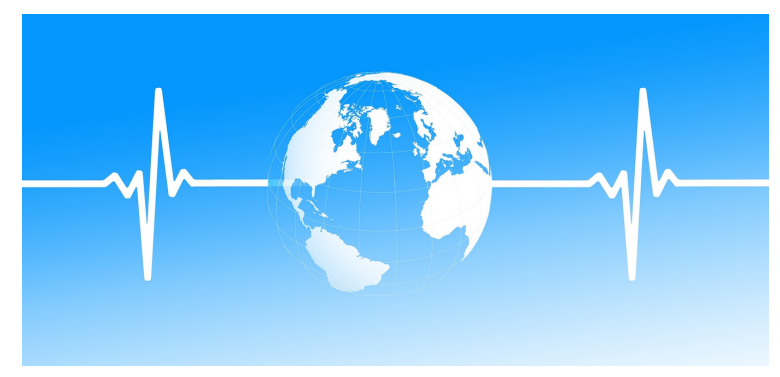

Figure 1: Salute pubblica (Pixabay)

Da un punto di vista operativo, generalmente, il procedimento può essere suddiviso in 5 fasi:

- SCREENING: trattasi di una fase preliminare in cui si decide se effettuare o meno la Valutazione di Impatto sulla Salute;

- SCOOPING: in questa fase si procede con la caratterizzazione dell'area interessata, si identificano i fattori di rischio, si effettua una valutazione dello stato di salute ante-operam della popolazione interessata e si procede con la scelta degli indicatori sanitari e delle figure professionali da coinvolgere;

- ASSESSMENT: in questa fase si vanno a quantificare i potenziali impatti sulla salute, definendo anche la loro relativa importanza in termini di magnitudo e probabilità;

- REPORTING: fase in cui si va a redigere un rapporto tecnico contenente misure atte a ridurre gli impatti involontari sull'ambiente e sulla salute umana dipendenti dalla realizzazione dell'opera analizzata;

- MONITORING: fase finale in cui si va a definire un piano di monitoraggio ambientale e sanitario per la verifica delle valutazioni condotte $^{7}$.

Comunque sia, la Valutazione di Impatto sulla Salute può essere essenzialmente di 3 tipi: prospettica, trasversale, retrospettiva ${ }^{8}$. 
Si parla di VIS prospettica quando ci collochiamo nella fase di progettazione e/o programmazione. La valutazione, in questo caso, ha lo scopo di accertare i potenziali impatti del progetto sulla salute, in modo da poter incidere sulle scelte finali ${ }^{9}$. Con una VIS trasversale si accertano, invece, gli impatti sulla salute durante la realizzazione dell'opera o del piano in oggetto, al fine di monitorare le trasformazioni sulla salute e sui suoi determinanti ${ }^{10}$. II ricorso ad una VIS retrospettiva permette, infine, di accertare gli impatti sanitari che si verificano a valle della realizzazione di un'opera o un piano ${ }^{11}$.

\section{Caso studio}

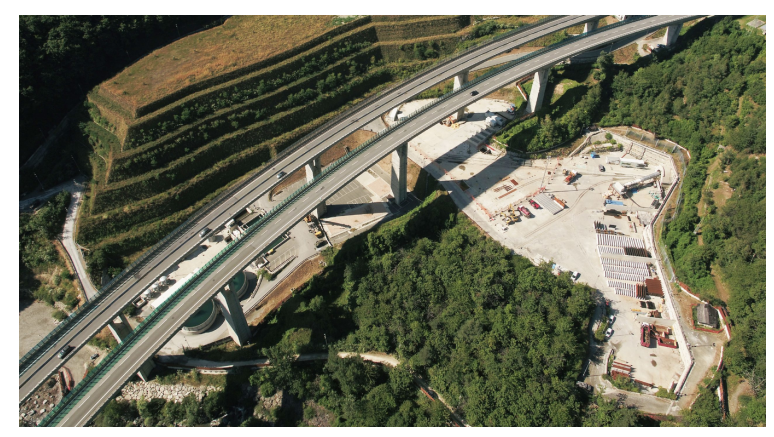

Figure 2: Vista dall'alto del cantiere (TELT)

Il caso studio preso in considerazione è quello di una galleria geognostica per la quale è stata condotta una Valutazione di Impatto sulla Salute di tipo trasversale.

L'opera, una galleria di circa $7 \mathrm{~km}$ di lunghezza e 6,30 $\mathrm{m}$ di diametro, è un tunnel esplorativo che in fase preliminare ha permesso di conoscere meglio la struttura geologica del massiccio che sarà poi attraversato dal tunnel principale, durante la realizzazione di quest'ultimo permetterà di accedere ai cantieri sotterranei dello stesso e nella fase di esercizio fungerà da tunnel di servizio per la manutenzione e come eventuale via di transito per i mezzi di soccorso ${ }^{12}$.

Già prima dell'inizio dei lavori, avvenuto nel gennaio 2013, la società committente aveva avviato nel 2012, in ottemperanza a precise richieste degli organi di controllo, un piano di monitoraggio ambientale e sanitario che ha previsto l'individuazione di circa 40 stazioni di monitoraggio, situate sia all'interno del cantiere, che nelle aree circostanti e nei comuni limitrofi, che in totale hanno prodotto oltre 60.000 misurazioni ${ }^{13}$. 
Tali misurazioni, la cui veridicità è stata comunque poi verificata e validata anche dall'ARPA di competenza, hanno riguardato, relativamente alle componenti ambientali, il clima, la concentrazione di amianto e di particolato nell'aria, la qualità dell'aria, i valori di rumore e di vibrazione, le radiazioni e la qualità delle acque, mentre per quanto riguarda i dati sanitari, sono stati presi in considerazione soprattutto gli indici di mortalità e di ricovero per le diverse patologie, per i diversi comuni monitorati ${ }^{14}$.

I dati raccolti durante la fase ante-operam del periodo di monitoraggio, integrati con altri già disponibili relativi a vecchie misurazioni, sono stati poi utilizzati per la costruzione del quadro di riferimento della salute del territorio; questi sono stati a loro volta confrontati con altri dati, raccolti durante la fase di realizzazione dell'opera conclusasi nel gennaio del 2017, basandosi sulle misurazioni effettuate dalle diverse stazioni e sulle informazioni desunte dalle varie fonti istituzionali ${ }^{15}$.

Da tale confronto emerge che, da un punto di vista dello stato di salute dell'ambiente, non si sono rilevati superamenti delle soglie limite disposte dalla normativa per le diverse componenti analizzate, eccezion fatta per la concentrazione di amianto e per le vibrazioni, che in tutto il periodo di monitoraggio hanno superato una sola volta il valore limite. In entrambi i casi però, da analisi più approfondite, è emerso che tali superamenti non erano legati alle attività di cantiere. Per quanto riguarda invece lo stato di salute della popolazione, dallo studio dei dati reperiti, si evince che $\mathrm{i}$ dati sanitari dei comuni monitorati, relativi alla fase ante-operam, sono pressoché allineati con quelli complessivi della regione e che, allo stesso modo, i dati relativi alla fase di realizzazione presentano sì una variazione degli indicatori di rischio di mortalità, che però rientra all'interno di quella che può essere definita come la naturale variabilità statistica del panorama di rischio regionale; lo stesso dicasi per i tassi di ricovero ${ }^{16}$.

In conclusione, si può affermare che la valutazione dei dati relativi ai fattori di pressione ambientale non ha segnalato alcun impatto dell'opera e che, lo stato di salute della popolazione è risultato pressoché simile, sia nella fase ante-operam che in fase di realizzazione, a quello complessivo della regione ${ }^{17}$.

\section{Conclusioni}

In definitiva, possiamo quindi sicuramente affermare che la VIS pone al centro dell'attenzione la protezione e la promozione della salute della popolazione, affinché le scelte garantiscano il benessere complessivo degli individui, delle comunità e la sostenibilità del loro ambiente. Altrettanto sicuramente possiamo affermare che questa si basa su determinati principi fondamentali, quali: inclusività, intesa nel senso di consentire agli stakeholder la partecipazione al processo valutativo; equità, nella distribuzione dei 
benefici e degli impatti legati all'opera e giustizia, ossia giusta considerazione di tutti i gruppi e dei loro reali bisogni di salute e benessere.

\section{References}

1.Ballarini, A. et al.. La valutazione di Impatto sulla Salute, un nuovo strumento a supporto delle decisioni. (2010).

2.Corpuz, M. V. A. et al.. Viruses in wastewater: occurrence abundance and detection methods. Science of The Total Environment 745, 140910 (2020).

3.Simeoni, T. V. Valutazione di Impatto Sanitario (V.I.S.). 12 domande (e risposte) chiave per capire la VIS. http://www.arpa.marche. it/images/epidemiologia/2013_esp/2013_02_vis/2013_vis.pdf (2013).

4.Benedetto, A. D. et al.. Valutazione di Impatto sulla Salute, Linee guida per proponenti e valutatori. (2016).

5.Naddeo, V. \& Korshin, G. Water energy and waste: The great European deal for the environment. Science of The Total Environment 764, 142911 (2021).

6.Naddeo, V. Development of environmental biotechnology and control of emerging biological contaminants: the grand challenge for a sustainable future. Water Environment Research 92, 1246-1248 (2020).

7.Musmeci, L. \& Soggiu, M. E. Linee guida per la Valutazione di Impatto Sanitario (VIS) (Legge 221/2015, art. 9). Roma: Istituto Superiore di Sanità (2017).

8.Naddeo, V., Balakrishnan, M. \& Choo, K. H. Frontiers in Water-EnergyNexus-Nature-Based Solutions Advanced Technologies and Best Practices for Environmental Sustainability. (Springer International Publishing, 2020). doi:10.1007/978-3-030-13068-8.

9.Naddeo, V., Belgiorno, V., Zarra, T. \& Scannapieco, D. Dynamic and embedded evaluation procedure for strategic environmental assessment. Land Use Policy 31, 605-612 (2013).

10.Scannapieco, D., Naddeo, V. \& Belgiorno, V. Sustainable power plants: A support tool for the analysis of alternatives. Land Use Policy 36, 478-484 (2014).

11.Nesticò, A., Elia, C. \& Naddeo, V. Sustainability of urban regeneration projects: Novel selection model based on analytic network process and zero-one goal programming. Land Use Policy 99, 104831 (2020). 
12.Oliva, G. et al.. Next-generation of instrumental odour monitoring system (IOMS) for the gaseous emissions control in complex industrial plants. Chemosphere 271, 129768 (2021).

13.Zarra, T., Reiser, M., Naddeo, V., Belgiorno, V. \& Kranert, M. A comparative and Critical Evaluation of Different Sampling Materials in the Measurement of OdouConcentration by Dynamic Olfactometry. CHEMICAL ENGINEERING TRANSACTIONS 30, (2012).

14.Naddeo, V., Zarra, T., Giuliani, S. \& Belgiorno, V. Odour Impact Assessment in Industrial Areas. CHEMICAL ENGINEERING TRANSACTIONS 30, (2012).

15.Giuliani, S. et al.. An Alternative Approach of the E-Nose Training Phase in Odour Impact Assessment. CHEMICAL ENGINEERING TRANSACTIONS 30, (2012).

16.Zarra, T., Naddeo, V., Giuliani, S. \& Belgiorno, V. Optimization of Field Inspection Method for Odour Impact Assessment. CHEMICAL ENGINEERING TRANSACTIONS 23, (2010).

17.Pira, E. \& Virano, M. Galleria geognostica di Chiomonte, Valutazione di impatto sulla Salute. https://www. comune. chiomonte.to.it/wpcontent/uploads/2017/05/Valutazione-Impatto-sulla-salute_.pdf (2017). 